Annuaire suisse de politique de développement

18 | 1999

La Suisse et l'action humanitaire

\title{
6. Culture et science
}

Catherine Schümperli Younossian et Thierry A. Freyvogel

\section{CpenEdition}

\section{Journals}

Édition électronique

URL : http://journals.openedition.org/aspd/684

DOI : 10.4000/aspd.684

ISSN : 1663-9669

\section{Éditeur}

Institut de hautes études internationales et du développement

\section{Édition imprimée}

Date de publication : 1 janvier 1999

Pagination : 195-197

ISSN : 1660-5934

\section{Référence électronique}

Catherine Schümperli Younossian et Thierry A. Freyvogel, « 6. Culture et science », Annuaire suisse de politique de développement [En ligne], 18 | 1999, mis en ligne le 17 juillet 2012, consulté le 08 septembre 2020. URL : http://journals.openedition.org/aspd/684 ; DOI : https://doi.org/10.4000/aspd.684 


\section{CULTURE ET SCIENCE*}

\subsection{RÉGLEMENTATION DU COMMERCE DE L'ART EN SUISSE}

Au début des années 90, la Suisse est mise sous pression et accusée d'être le lieu de transit d'un commerce illégal de biens culturels étrangers. Pour sa part, le Conseil fédéral reconnaît le besoin de réglementation, constatant que la Suisse ne dispose pas, contrairement à d'autres pays européens, de lignes directrices réglant le contrôle du commerce illicite.

Au niveau international, il existe deux conventions traitant de cette problématique: la Convention de l'UNESCO du 14 novembre 1970 concernant les mesures à prendre pour empêcher l'importation, l'exportation et le transfert de propriété illicites des biens culturels et la Convention d'Unidroit du 24 juin 1995 sur les biens culturels volés et illicitement exportés. La Suisse n'a ratifié aucune des deux conventions; elle a cependant signé la convention Unidroit en juin 1996. A cette même date, le Conseil fédéral a constitué un groupe de travail chargé d'éclaircir un certain nombre de questions juridiques soulevés par les deux conventions.

Le groupe de travail interdépartemental a rédigé un rapport, «Transfert international de biens culturels ${ }^{1}$, auquel ont été associés d'éminents juristes. Rendu public en août 1998, le rapport présente de manière exhaustive les différents outils juridiques pertinents pour réglementer le commerce de biens culturels (conventions Unidroit et UNESCO, ainsi qu'un règlement et une directive de la Communauté européenne). Dans ses conclusions, le rapport souligne «qu'Unidroit est un instrument permettant de faire face efficacement aux problèmes qui se posent en matière de transfert international de biens culturels. Une ratification d'UNESCO se justifie en particulier en ceci que cette convention crée un cadre multilatéral pour la coopération internationale en matière de protection internationale de biens culturels». L'option de la ratification des deux conventions ainsi que la rédaction d'une loi d'application en droit suisse sont retenues par le groupe de travail.

ASTM 1997, partie «Analyses et positions», pp. 271-282: l'article de C. Schümperli Younossian «Commerce, importation et exportation de biens culturels: état de la réglementation en Suisse» présente les deux conventions et dresse une chronologie des différentes étapes de la discussion en Suisse.

Le Conseil fédéral n'a toutefois pas suivi les recommandations du groupe d'experts puisqu'il a chargé le Département de l'intérieur de préparer un message concernant la ratification de la convention d'UNESCO de 1970, message qui doit également proposer les modifications du droit national résultant de la ratification de cette convention. Quant à la convention d'Unidroit, le Conseil fédéral consi-

* Partie 6.1.: par Catherine Schümperli Younossian, chargée de recherche à l'IUED.

Partie 6.2.: par Thierry A. Freyvogel, Commission suisse pour le partenariat scientifique avec les pays en développement (KFPE), Berne.

1. Office fédéral de la culture, rapport du groupe de travail «Transfert international de biens culturels», OFC, Berne, septembre 1998. Disponible à l'Office central des imprimés et du matériel, 3000 Berne. 
dère que cette décision est prématurée; mais à toutefois chargé le DFI «de poursuivre l'examen de l'évolution internationale et, le moment venu, de faire des propositions au Conseil fédéral».

\section{SOURCES}

Office fédéral de la culture, rapport du groupe de travail «Transfert international de biens culturels», OFC, Berne, septembre 1998.

Le Temps, «Unidroit s'efface, le marché de l'art respire», 27.8.98.

Neue Zürcher Zeitung, Andrea Rasher, «Besserer Schutz für Museen und Sammler. Die Unidroit-Konvention: ein Kompromiss, der allen dient», 13.10.98.

INDICATION BIBLIOGRAPHIQUE

Watson Peter, L'affaire Sotheby's. Enquête d'un scandale, Belfond, Paris, 1997.

\subsection{PARTENARIATS SCIENTIFIQUES AVEC LES PAYS EN DÉVELOPPEMENT}

En mars 1996, la Commission suisse pour le partenariat scientifique avec les pays en développement (KFPE) a organisé à Berne une conférence de plusieurs jours qui s'intitulait «International Conference on Scientific Research Partnership for Sustainable Development: North-South and South-South Dimensions». Mise sur pied sous l'égide de l'Association européenne des instituts de recherche et de formation en matière de développement $(\mathrm{EADI})^{2}$, la conférence avait pour thème principal la stratégie suisse pour l'encouragement de la recherche dans les pays en développement. Cette stratégie avait été élaborée entre 1990 et 1993 par un groupe interdisciplinaire de l'Académie suisse des sciences naturelles $(\mathrm{ASSN})^{3}$, et la Conférence des académies scientifiques suisses (CASS) a ensuite créé (en 1994) la KFPE, qui est chargée de l'appliquer.

Pour les organisateurs de la conférence, conclure des partenariats scientifiques constitue un excellent moyen de promouvoir la recherche dans les pays en développement. Dans la mesure du possible, de tels partenariats devraient toutefois respecter un code de conduite - une demande exprimée par les participants à la conférence. En 1998, après deux années de travail, la KFPE a donc présenté son Guide du partenariat scientifique avec les pays en développement - 11 principes $^{4}$.

Ce guide sera diffusé en Suisse et à l'étranger, dans les milieux scientifiques, politiques et économiques, ainsi qu'auprès des organismes et des personnes intéressés. Relevons que des représentants du Groupement de la science et de la recherche (GSR), de la Direction du développement et de la coopération (DDC) et du Fonds national suisse de la recherche scientifique (FNRS) ont aussi participé à la présentation de ce guide aux médias. Ces instances souhaitent en effet que les rédacteurs du «Message concernant la promotion de l'éducation, de la recherche et de la technologie pour les années 2000 à 2003» tiennent compte de l'importance et de l'urgence d'une promotion de la recherche dans les pays en développement et en transition.

2. Rapport de D. Maselli et B. de Sottas (eds.), Research Partnerships for Common Concerns, LIT Verlag, Hamburg, 1996.

3. Stratégie suisse pour l'encouragement de la recherche dans les pays en développement, 1993 (2 $2^{\circ}$ édition 1996), disponible auprès du secrétariat de la KFPE, Bärenplatz 2, CH-3011 Berne.

4. Ce guide peut être obtenu en allemand, en anglais et en français auprès du secrétariat de la KFPE. Les versions espagnole et portugaise sont en préparation. 
Ces dix dernières années, la Suisse s'est de plus en plus attachée à encourager la recherche dans les pays en développement. Les 11 principes esquissés dans le guide ont ainsi déjà fait leurs preuves au Centre suisse de recherches scientifiques en Côte d'Ivoire et à l'Ifakara Health Research and Development Centre en Tanzanie. Ils sont également appliqués dans la majorité des projets du module 7 «Développement et environnement» du programme national prioritaire «Environnement», qui sont cofinancés par le FNRS et la DDC. Ces deux organismes souhaitent d'ailleurs poursuivre leur collaboration pour encourager la recherche dans le domaine du développement. Pour sa part, la Commission fédérale des bourses pour étudiants étrangers désire tenir compte de l'orientation à long terme de la coopération suisse au développement dans l'octroi de bourses aux étudiants des pays en développement.

Avec le soutien de diverses institutions, la KFPE a ainsi développé et promu l'idée de partenariats scientifiques de la Suisse avec des pays en développement et en transition ${ }^{5}$. Mais il reste encore beaucoup à faire car ni les milieux politiques et économiques, ni même les milieux scientifiques n'ont encore réellement pris conscience de tout ce que ces partenariats peuvent apporter à l'enseignement et à la recherche en Suisse. La CASS a dès lors décidé de faire de la KFPE une commission permanente, dont les nouveaux statuts sont en préparation.

5. Les rapports annuels peuvent être consultés sur le site http://www.kfpe.unibe.ch 